\title{
An upper bound for the Waring rank of a form
}

\author{
JOACHIM JELISIEJEW
}

\begin{abstract}
In this paper we introduce the open Waring rank of a form of degree $d$ in $n$ variables and prove that this rank is bounded from above by

$$
\left(\begin{array}{c}
n+d-2 \\
d-1
\end{array}\right)-\left(\begin{array}{c}
n+d-6 \\
d-3
\end{array}\right)
$$

whenever $n, d \geq 3$. This proves the same upper bound for the classical Waring rank of a form, improving the result of Białynicki-Birula and Schinzel (see [4]) and giving, as far as we know, the best upper bound known.
\end{abstract}

Mathematics Subject Classification (2010). 13P05.

Keywords. Sums of powers of linear polynomials, Waring problem, Waring rank, Open Waring rank.

1. Introduction. The Waring rank of a homogeneous polynomial $F$ of degree $d$ is the minimal number $r$ such that there exists a presentation $F=\sum_{i=1}^{r} l_{i}^{d}$, where $l_{i}$ are linear forms. It is a classical problem to find the maximal possible Waring rank of $F$ for fixed $d$ and the number $n$ of variables. For $n=2$ it was solved by Sylvester, see [13] and [7], but in general it remains open. The problem has drawn much attention after Alexander and Hirschowitz computed the Waring rank of a general homogeneous polynomial, see [1]. For an account of what is known about the rank and for recent results, see $[3,4,6,10]$,

Supported by the project "Secant varieties, computational complexity, and toric degenerations" realised within the Homing Plus programme of Foundation for Polish Science, co-financed from European Union, Regional Development Fund. This paper is a part of "Computational complexity, generalised Waring type problems and tensor decompositions" project within "Canaletto", the executive program for scientific and technological cooperation between Italy and Poland, 2013-2015. 
[11, Chap 9], [12] and references therein. See especially the recent paper by Brekherman and Teitler [5], which improves our bound for large $n$ and $d$.

In [4] the authors introduce a stronger version of the Waring rank of a homogeneous polynomial (see Remark 3 for the precise definition) and give an upper bound $\left(\begin{array}{c}n+d-2 \\ d-1\end{array}\right)$ for the rank of a form of degree $d$ in $n$ variables. In this article we introduce an even slightly stronger version of the rank and improve the upper bound to

$$
\left(\begin{array}{c}
n+d-2 \\
d-1
\end{array}\right)-\left(\begin{array}{c}
n+d-6 \\
d-3
\end{array}\right)
$$

which proves the same bound for the classical Waring rank. This bound is sharp for $n=d=3$, see e.g. [10, Section 2]. It is not sharp for $n=3$ and $d=4$, see [2, Introduction].

Notation. Let $k$ be an algebraically closed field of characteristic zero and $S=$ $k\left[x_{1}, \ldots, x_{n}\right]$ be a polynomial ring. We will often think of $S_{1}$ as an affine space; in this spirit let $V \subsetneq S_{1}$ be a Zariski closed subset. Let $S^{*}=k\left[\frac{\partial}{\partial x_{1}}, \ldots, \frac{\partial}{\partial x_{n}}\right]$ be the ring of differential operators with its usual action on $S$, which is denoted by $(-)\lrcorner(-): S^{*} \otimes S \rightarrow S$. For $F \in S_{d}$ by $F^{\perp} \subseteq S^{*}$ we denote the annihilator of $F$ with respect to this action. Throughout the paper we consider only homogeneous polynomials in $S$. We use the terms homogeneous polynomial and form interchangeably.

Definition 1. A form $F \in S$ essentially depends on $n$ variables if it cannot be written using less than $n$ variables after a linear change of coordinates.

Definition 2. For a form $F \in S$ of degree $d$ and $V \subseteq S_{1}$ defined as above, let $m=\operatorname{Ork}(F, V)$ be the minimal natural number such that there exists a presentation

$$
F=\sum_{i=1}^{m} l_{i}^{d}, \quad \text { where } l_{i} \notin V,
$$

or $\operatorname{Ork}(F, V)=\infty$ if such presentation does not exist. Define the open Waring rank of $F$ by

$$
\operatorname{Ork}(F):=\sup \left\{\operatorname{Ork}(F, V) \mid V \subsetneq S_{1} \text { homogeneous and Zariski closed }\right\} .
$$

Finally take

$$
\operatorname{Ork}(n, d):=\sup \left\{\operatorname{Ork}(F) \mid F \in S_{d} \text { essentially depends on } n \text { variables }\right\} \text {. }
$$

Remark 3. The classical Waring rank of a form $F$ is equal to Ork $(F, \emptyset)$. The rank defined in [4] is similar to the one defined above, the difference is that the authors consider only subsets $V \subsetneq S_{1}$ which are finite sums of hyperplanes through the origin:

$$
\begin{aligned}
& S(n, d):=\sup \left\{\operatorname{Ork}(F, V) \mid V \subsetneq S_{1}\right. \text { is a finite sum } \\
& \quad \text { of hyperplanes containing the origin, } \\
& \left.F \in S_{d} \text { essentially depends on } n \text { variables }\right\} .
\end{aligned}
$$

Thus we have an inequality $\operatorname{Or} k(n, d) \geq S(n, d)$. 
In [4] the key point is the inequality

$$
S(n, d) \leq S(n, d-1)+S(n-1, d),
$$

for $n, d \geq 3$, which gives a recursive step in the proof of the upper bound $S(n, d) \leq\left(\begin{array}{c}n+d-2 \\ d-1\end{array}\right)$. The base cases of the recursion are the equalities

$$
S(2, d)=d \quad \text { and } \quad S(n, 2)=n,
$$

so the smallest case where the obtained upper bound may be sharp is $S(3,3)$; the article [4] gives the bound $S(3,3) \leq 6$. We prove the inequality (1) together with the base cases (2) for the open rank, thus obtaining a bound

$$
\operatorname{Ork}(n, d) \leq\left(\begin{array}{c}
n+d-2 \\
d-1
\end{array}\right) .
$$

Next we prove that $\operatorname{Ork}(3,3)=5$, which improves the upper bound to

$$
\operatorname{Ork}(n, d) \leq\left(\begin{array}{c}
n+d-2 \\
d-1
\end{array}\right)-\left(\begin{array}{c}
n+d-6 \\
d-3
\end{array}\right) .
$$

In the proof we will both adopt (in Lemma 13) and reference the ideas from the Master thesis of Johannes Kleppe [10].

The paper is divided into two sections, preceded by a preliminary part. In the first section we prove:

Theorem 4. Let $n, d \geq 2$ be integers. We have equations Ork $(2, d)=d$ and $\operatorname{Ork}(n, 2)=n$. Moreover,

$$
\operatorname{Ork}(n, d) \leq \operatorname{Ork}(n-1, d)+\operatorname{Ork}(n, d-1)
$$

for every $n, d \geq 3$.

The proof is a copy of the proof of (1) and (2) from [4]. Unfortunately the proof given there is, formally, just a special case of the proof required and moreover Białynicki-Birula and Schinzel are also concerned with non-homogeneous polynomials, which makes their proof more complicated.

In the second part, we prove the following theorem, with an immediate corollary bounding the open Waring rank:

Theorem 5. Ork $(3,3)=5$.

Corollary 6. Let $n, d \geq 3$ be integers, then

$$
\operatorname{Ork}(n, d) \leq\left(\begin{array}{c}
n+d-2 \\
d-1
\end{array}\right)-\left(\begin{array}{c}
n+d-6 \\
d-3
\end{array}\right) .
$$

2. Preliminaries. Let us recall a special case of so-called Apolarity Lemma:

Lemma 7. If $F \in S_{d}$ and $\bigcap_{i=1}^{k} \mathfrak{m}_{i} \subseteq F^{\perp}$, where $\mathfrak{m}_{i}$ are homogeneous ideals of distinct points $\left[l_{i}\right] \in \mathbb{P} S_{1}$, then

$$
F \in\left\langle l_{1}^{d}, l_{2}^{d}, \ldots, l_{k}^{d}\right\rangle .
$$

Proof. See [9, Lemma 1.15].

Another lemma, whose proof can be found in [10], is concerned with linear systems obtained from the apolar ideal of a form: 
Lemma 8. Let $F$ be a form in $S_{d}$. Choose $e \leq d$ and consider $\mathcal{L}=\left(F^{\perp}\right)_{e}$ as a linear system on $\mathbb{P} S_{1}$. A point $[l] \in \mathbb{P} S_{1}$ is a base point of $\mathcal{L}$ if and only if there exists a differential $\partial \in S_{d-e}^{*}$ such that $\partial F=l^{e}$.

Sketch of proof. Fix a point $[l] \in \mathbb{P} S_{1}$ with homogeneous ideal $\mathfrak{m}_{l}$. The point $[l]$ is a base point of $\mathcal{L}$ iff $\left(\mathfrak{m}_{l}\right)_{e} \supseteq\left(F^{\perp}\right)_{e}=\bigcap\left\{(\partial F)_{e}^{\perp} \mid \partial \in S_{d-e}^{*}\right\}$ iff there exists $\partial \in S_{d-e}^{*}$ such that $l^{e}=\partial F$.

Corollary 9. Fix $d \geq e \geq 1$. Denote by Ess $s_{\text {,e }}$ the set of forms $F \in S_{d}$ such that no nonzero element of $S_{e}^{*}$ annihilates $F$. The set of $F \in E s s_{d, e}$ such that $\left(F^{\perp}\right)_{d-e}$ has a base point in $\mathbb{P} S_{1}$ is closed in Ess $s_{d, e}$.

Proof. Note that $E s s_{d, e}$ is Zariski open in $S_{d}$. Denote by $W$ the subset of forms $F \in E s s_{d, e}$ such that $\left(F^{\perp}\right)_{d-e}$ has a base point in $\mathbb{P} S_{1}$. Consider the closed subvariety

$$
\left\{(F,[\partial],[l]) \in E s s_{d, e} \times \mathbb{P} S_{e}^{*} \times \mathbb{P} S_{1} \mid l^{d-e} \text { and } \partial F \text { are linearly dependent }\right\} .
$$

The projection to the first coordinate gives the set of forms $F \in E s s_{d, e}$ such that there exist $\partial \in S_{e}^{*}, l \in S_{1}$, and $\lambda, \lambda^{\prime} \in k$, not both equal zero, satisfying $\lambda l^{d-e}=\lambda^{\prime} \partial F$. As $l^{d-e} \neq 0$ and $\partial F \neq 0$ from the definition of $E s s_{d, e}$, we have $\lambda \lambda^{\prime} \neq 0$, which is equivalent, by Lemma 8 , to $F \in W$.

3. Proof of Theorem 4. The proof will be divided into three independent lemmas.

Lemma 10. Let $d \geq 2$ be an integer, then $\operatorname{Ork}(2, d)=d$.

Proof. Denote $S=k\left[x_{1}, x_{2}\right]$. Let $F \in S_{d}$ be a form which essentially depends on two variables and $V \subsetneq S_{1}$ be homogeneous and Zariski-closed. We would like to show that $\operatorname{Ork}(F, V) \leq d$. The proof resembles the proof of a standard fact that the Waring rank of $F$ is at most $d$, however we must also take into account $V$.

It is a classical result by Sylvester that $F^{\perp}$ is a complete intersection generated by elements of degrees $d_{1}$ and $d_{2}$ such that $d_{1}+d_{2}=d+2$. If $\min \left(d_{1}, d_{2}\right)=1$, then $F$ does not essentially depend on two variables. Thus $\min \left(d_{1}, d_{2}\right) \geq 2$ and $\max \left(d_{1}, d_{2}\right) \leq d$. In particular the linear system $F_{d}^{\perp}$ on $\mathbb{P} S_{1}$ is base point free. By the Bertini Theorem [8, Thm III.10.9], a general element $D$ of $F_{d}^{\perp}$ is smooth and does not intersect $V$. The zero set of $D$ is a sum of $d$ points, which, by Lemma 7 , gives a required presentation of $F$. Thus $\operatorname{Ork}(F, V) \leq d$ and $\operatorname{Ork}(2, d) \leq d$.

The form $x_{1}^{d-1} x_{2}$ has Waring rank $d$, thus its open rank is at least $d$, see Remark 3, therefore $\operatorname{Ork}(2, d) \geq d$.

Lemma 11. Let $n \geq 1$ be an integer, then $\operatorname{Ork}(n, 2)=n$.

Proof. The inequality $\operatorname{Ork}(n, 2) \geq n$ is trivial because the sum of less than $n$ squares does not essentially depend on $n$ variables. We prove the other inequality by induction on $n$, the base being clear. Let $n \geq 2$. Take a form $F \in S_{2}$ which essentially depends on $n$ variables and $V \subsetneq S_{1}$ homogeneous and Zariski-closed. 
Consider $S_{1}^{*}$ as an affine space. For $\partial \in S_{1}^{*}$ the condition $\left.\partial^{2}\right\lrcorner F=0$ is Zariski-closed. Take any $\alpha \in S_{1}^{*}$ such that $\left.\alpha^{2}\right\lrcorner F \neq 0$ and $V(\alpha) \not \subset V$. Let

$$
F^{\prime}=F-\frac{(\alpha\lrcorner F)^{2}}{\left.2 \cdot \alpha^{2}\right\lrcorner F},
$$

then $\alpha\lrcorner F^{\prime}=0$, thus $F^{\prime}$ may be written, after a linear change of coordinates, in $n-1$ variables $x_{1}^{\prime}, \ldots, x_{n-1}^{\prime}$ such that $\left.\alpha\right\lrcorner x_{1}^{\prime}=0$. From the definition of $F^{\prime}$, it follows that $F$ may be written using one more variable than $F^{\prime}$, thus $F^{\prime}$ essentially depends on $n-1$ variables. Furthermore $V^{\prime}=V \cap V(\alpha) \neq V(\alpha)$ is a homogeneous Zariski-closed set, so that $\operatorname{Ork}\left(F^{\prime}, V^{\prime}\right) \leq n-1$ by induction, and we obtain $\operatorname{Ork}(F, V) \leq \operatorname{Ork}\left(F^{\prime}, V^{\prime}\right)+1 \leq n$.

Lemma 12. Let $n, d \geq 3$ be integers, then

$$
\operatorname{Ork}(n, d) \leq \operatorname{Ork}(n-1, d)+\operatorname{Ork}(n, d-1) .
$$

Proof. Take $F \in S_{d}$ which essentially depends on $n$ variables and $V \subsetneq S_{1}$ homogeneous and Zariski-closed. Take $\alpha \in S_{1}^{*}$ such that $V(\alpha) \not \subset V$ and $F^{\prime}=$ $\alpha\lrcorner F$ essentially depends on $n$ variables (these are open non-empty conditions). The form $F^{\prime}$ has a presentation

$$
F^{\prime}=\sum_{i=1}^{m} l_{i}^{d-1},
$$

where $m=\operatorname{Ork}\left(F^{\prime}, V \cup V(\alpha)\right)$ and $l_{i} \notin V \cup V(\alpha)$. Note that $l_{i} \notin V(\alpha)$ is equivalent to $\alpha\lrcorner l_{i} \neq 0$. Take

$$
F_{1}=\sum_{i=1}^{m} \alpha_{i} \cdot l_{i}^{d}
$$

where $\left.\alpha_{i}=(d \cdot \alpha\lrcorner l_{i}\right)^{-1}$, then $\left.\alpha\right\lrcorner\left(F-F_{1}\right)=0$. Let $T \subseteq\{1,2, \ldots, m\}$ be a minimal set of indexes such that there exists $0 \neq \beta=\beta_{T} \in S_{1}^{*}$ such that

1. $V(\beta) \not \subset V$,

2. $F_{2}:=F-\sum_{i \in T} \alpha_{i} \cdot l_{i}^{d}$ is annihilated by $\beta$,

(the set $T=\{1, \ldots, m\}$ with $\alpha=\beta_{T}$ satisfies the above hypotheses except, perhaps, minimality). We claim that the form $F_{2}$ obtained from a minimal $T$ essentially depends on $n-1$ variables. If this is not the case, then we take $i \in T$ such that $F_{2}+\alpha_{i} \cdot l_{i}^{d}$ essentially depends on more variables than $F_{2}$. The space $\left(F_{2}\right)_{1}^{\perp}$ is at least two-dimensional, thus its intersection with $\left(l_{i}^{d}\right)_{1}^{\perp}$ contains a non-zero element $\beta^{\prime}$. Since $l_{i} \in V\left(\beta^{\prime}\right) \backslash V$, we have $V\left(\beta^{\prime}\right) \not \subset V$ and the set $T^{\prime}:=T \backslash\{i\}$ satisfies the above conditions. This contradicts the minimality of $T$.

Since $F_{2} \in k\left[x_{1}, \ldots, x_{n}\right]$ essentially depends on $n-1$ variables lying in $V(\beta)$ and $V \cap V(\beta) \neq V(\beta)$, the form $F_{2}$ may be written using at most $m_{2} \leq \operatorname{Ork}(n-1, d)$ powers of linear forms taken from outside $V$. The field $k$ is algebraically closed, thus (3) shows that $F=\left(F-F_{2}\right)+F_{2}$ may be written using at most $m+m_{2} \leq \operatorname{Ork}(n, d-1)+\operatorname{Ork}(n-1, d)$ powers of linear forms taken from outside $V$. 
4. Proof of Theorem 5. From now on $n=3$, i.e. $S:=k\left[x_{1}, x_{2}, x_{3}\right]$. First we deal with the majority of forms, using the following lemma:

Lemma 13. Let $F \in S_{3}$ be such that $V\left(\left(F^{\perp}\right)_{2}\right) \subseteq \mathbb{P} S_{1}$ is an empty set. Then $\operatorname{Ork}(F, V) \leq 4$ for any homogeneous closed $V \subsetneq S_{1}$.

Proof. Let $V^{\prime} \subseteq \mathbb{P} S_{1}$ be the image of $V \backslash\{0\}$, then $V^{\prime}$ is closed and not equal to $\mathbb{P} S_{1}$.

By Bertini's theorem [8, Thm III.10.9] applied to the base point free linear system $\left(F^{\perp}\right)_{2}$ on $\mathbb{P} S_{1}$, we see that the general element $D$ of this system is smooth. At the same time, a general element $D$ intersects $V^{\prime}$ properly, i.e. $\operatorname{dim} V(D) \cap V^{\prime}<\operatorname{dim} V^{\prime}$. We choose $D_{0}$ satisfying both properties.

Restricting to $V\left(D_{0}\right)$ and using Bertini's theorem once more, we obtain an element $D_{1} \in\left(F^{\perp}\right)_{2}$ such that $V\left(D_{0}\right) \cap V\left(D_{1}\right)$ is smooth of dimension zero and $V\left(D_{0}\right) \cap V\left(D_{1}\right) \cap V^{\prime}$ is empty. From Lemma 7 it follows that

$$
F \in\left\langle l_{a_{1}}^{3}, l_{a_{2}}^{3}, l_{a_{3}}^{3}, l_{a_{4}}^{3}\right\rangle,
$$

where $\left\{a_{1}, a_{2}, a_{3}, a_{4}\right\}=V\left(D_{0}, D_{1}\right)$ so $\left\{a_{1}, a_{2}, a_{3}, a_{4}\right\} \cap V^{\prime}=\emptyset$.

Now we would like to show that the set of "bad forms", i.e. those which do not satisfy the assumptions of Lemma 13, is closed in the (open) set of all forms which essentially depend on three variables.

Corollary 14. Denote by Ess the (open) set of forms which essentially depend on three variables, and let $W \subseteq$ Ess be the subset consisting of forms such that $V\left(\left(F^{\perp}\right)_{2}\right) \subseteq \mathbb{P} S_{1}$ is not an empty set. Then $W$ is closed in Ess.

Proof. This follows from Corollary 9 applied to the case $d=3, e=1$.

Finally, we need an explicit characterisation of the "bad forms" due to Kleppe:

Proposition 15. Consider the set of forms $F \in S_{3}$ essentially dependent on three variables and such that $V\left(\left(F^{\perp}\right)_{2}\right) \subseteq \mathbb{P} S_{1}$ is not an empty set. Every element of this set is an image, under a linear change of basis in $S_{1}$, of one of the following forms

$$
x_{0} x_{1}^{2}+x_{1} x_{2}^{2} \quad \text { or } \quad x_{0}^{3}+g \text { where } g \in k\left[x_{1}, x_{2}\right]_{3} \text {. }
$$

Furthermore, the classical Waring rank of $x_{0} x_{1}^{2}+x_{1} x_{2}^{2}$ is five.

Proof. See [10, Theorem 2.3].

Now we are ready to prove Theorem 5 .

Proof of Theorem 5. By Proposition 15 it sufficies to prove Ork $(3,3) \leq 5$. Take a form $F \in S_{3}$ which essentially depends on three variables and a homogeneous closed subset $V \subsetneq \mathbb{A}^{3}$.

If $F$ satisfies the assumptions of Lemma 13, then $\operatorname{Ork}(F, V) \leq 4$ and we are done. Denote the set of the forms which essentially depend on three variables and satisfy the assumptions of Lemma 13 by $U$.

If $F \notin U$, then $F \in W$, where $W$ was defined in Corollary 14 . In this case we would like to find a linear form $l$ such that $F+l^{3} \in U$. After a linear 
change of coordinates we can assume $F$ is of the form from Lemma 15. For $x_{0} x_{1}^{2}+x_{1} x_{2}^{2}$ the form $l^{3}=\left(x_{0}+x_{1}\right)^{3}$ will do, and in the second case we can write $g=x_{1} x_{2}\left(x_{1}+a x_{2}\right)$ where $a \in k$, then $l^{3}=-\left(x_{0}+x_{2}\right)^{3}$ will do.

The set of forms which essentially depend on three variables is open in the set of all forms, and the set $U$ is open in this set by Corollary 14, so that $U$ is open in the set of all forms. We have just seen that $U$ has non-empty intersection with $\left\{F+l^{3}\right\}$, so $U \cap\left\{F+l^{3}\right\}$ is open in this set. Choosing $l \notin V$ such that $F+l^{3} \in U$, we get the required result.

Acknowledgements. The author is very grateful to Jarosław Buczyński for suggesting this research topic as well as his constant support and to Enrico Carlini for insisting on writing down this paper. The author thanks the referee for suggesting several improvements of the exposition.

Open Access. This article is distributed under the terms of the Creative Commons Attribution License which permits any use, distribution, and reproduction in any medium, provided the original author(s) and the source are credited.

\section{References}

[1] J. Alexander and A. Hirschowitz, Polynomial interpolation in several variables. J. Algebraic Geom. 4 (1995), 201-222.

[2] E. Ballico And A. De Paris, Generic Power Sum Decompositions and Bounds for the Waring Rank. arXiv:1312.3494, 2013.

[3] A. Bernardi, A. Gimigliano, and M. IdÀ, Computing symmetric rank for symmetric tensors. J. Symbolic Comput. 46 (2011), 34-53.

[4] A. Biąynicki-Birula And A. Schinzel, Representations of multivariate polynomials by sums of univariate polynomials in linear forms. Colloq. Math. 112 (2008), 201-233.

[5] G. Blekherman and Z. Teitler, On maximum, Typical, and Generic Ranks. arXiv:1402.2371, 2014.

[6] E. Carlini, M. V. Catalisano, and A. V. Geramita, The solution to the Waring problem for monomials and the sum of coprime monomials. J. Algebra 370 (2012), 5-14.

[7] G. Comas and M. Seiguer, On the rank of a binary form, Found. Comput. Math. 11 (2011), 65-78.

[8] R. Hartshorne, Algebraic geometry. Springer-Verlag, New York, 1977, Graduate Texts in Mathematics, No. 52.

[9] A. Iarrobino And V. Kanev, Power sums, Gorenstein algebras, and determinantal loci, volume 1721 of Lecture Notes in Mathematics. Springer-Verlag, Berlin, 1999. Appendix C by Iarrobino and Steven L. Kleiman.

[10] J. KLeppe, Representing a Homogeneous Polynomial as a Sum of Powers of Linear Forms. available at http://folk.uio.no/johannkl/kleppe-master.

[11] J. M. LAndsberg, Tensors: geometry and applications, volume 128 of Graduate Studies in Mathematics. American Mathematical Society, Providence, RI, 2012. 
[12] J. M. Landsberg and Z. Teitler, On the ranks and border ranks of symmetric tensors. Found. Comput. Math. 10 (2010), 339-366.

[13] J. J. Sylvester, Sur une extension d'un théorème de Clebsch relatif aux courbes du quatrième degré . Math. Acad. Sci. Paris 102 (1886), 1532-1534.

\section{JOACHIM JELISIEJEW}

Faculty of Mathematics, Informatics, and Mechanics, University of Warsaw,

Banacha 2, 02-097 Warsaw, Poland

e-mail: jjelisiejew@mimuw.edu.pl

Received: 19 October 2013 